\title{
THE ARCUATE FASCICULUS NETWORK AND VERBAL DEFICITS IN PSYCHOSIS
} precise neuroanatomical contributions are not fully understood. We investigated the arcuate fasciculus (AF) and its associated cortical regions to identify structural abnormalities contributing to these verbal impairments in early stages of psychotic illness.

Methods: Twenty-six individuals with recent-onset psychosis and 27 healthy controls underwent cognitive testing (MATRICS Consensus Cognitive Battery) and structural/diffusion-weighted MRI. Bilaterally, AF anisotropy and cortical thickness, surface area and volume of seven cortical regions were investigated in relation to VL and VF performance in both groups.

Results: Reduced right superior temporal gyrus surface area and volume related to better VF in controls. In psychosis, greater right pars opercularis volume and reduced left lateralization of this region related to better $\mathrm{VL}$, while greater right long AF fractional anisotropy and right pars orbitalis volume related to better VF, these findings not present in controls. Psychosis had reduced right pars orbitalis thickness compared to controls.

Conclusion: Anatomical substrates for normal processing of VL and VF appear altered in recent-onset psychosis. A possible aberrant role of the right hemisphere arcuate fasciculus and fronto-temporal cortical regions in psychosis may contribute to deficits in VL and VF.

Keywords

- Arcuate Fasciculus • Cortical Language Regions • Verbal Learning • Verbal Fluency - Psychosis • Structural MRI • Diffusion MRI • Lateralization

\section{Introduction}

Cognitive deficits are prevalent in early stages of psychosis, deficits of verbal cognition being the most consistent in the literature [1], which may also be susceptible to further deterioration longitudinally [2]. In a previous study, we identified verbal learning and verbal fluency to remain persistently impaired compared to other cognitive domains four years subsequent to a first episode of psychosis [3]. These verbal deficits have been linked to functional outcome [4] and positive symptoms of the disorder $[5,6]$ and it is therefore vital to understand the precise neuroanatomical contributions of these cognitive impairments to assist in early diagnosis and targeted therapeutic interventions. In schizophrenia, structural and functional abnormalities have been found in language related brain regions such as the arcuate fasciculus (AF) [7]. This white matter tract is strongly implicated in verbal abilities as lesions to the AF have been linked with disorders of language such as conduction aphasia [8]. However, its involvement in verbal impairments in psychosis is not yet certain. The classical model of the (AF), as identified by gross dissection studies, describes the AF as a long direct tract originating in the caudal temporal lobe including Wernicke's territory, sweeping around the insula and sylvian fissure and connecting with the posterior portion of the inferior frontal lobule, synonymous with Broca's regions. More recently, with the advent of modern diffusion tensor imaging (DTI) techniques, in vivo reconstruction of the arcuate fasciculus has revealed an additional two indirect tracts of the $\mathrm{AF}$, the anterior tract linking the inferior-parietal lobule (Geschwind's territory) to frontal regions and a posterior tract connecting Geschwind's territory with temporal regions [9] (Figure 1). Most DTI studies
Joanne P.M. Kenney ${ }^{1 *}$,

Genevieve McPhilemy',

Cathy Scanlon ${ }^{1}$

Pablo Najt ${ }^{1}$

Shane Mclnerney ${ }^{1,2}$,

Sophia Arndt',

Elisabeth Scherz',

Fintan Byrne',

Alexander Leemans ${ }^{3}$,

Ben Jeurissen ${ }^{4}$,

Brian Hallahan',

Colm McDonald',

Dara M.Cannon'

Centre for Neuroimaging \& Cognitive Genomics (NICOG), Clinical Neuroimaging Laboratory, NCBES Galway Neuroscience Centre, College of Medicine Nursing and Health Sciences, National University of Ireland Galway, H91 TK33, Galway, Ireland.

${ }^{2}$ Departments of Psychiatry, St Michaels Hospital \&

University of Toronto, Toronto, Canada

${ }^{3}$ Images Sciences Institute, University Medical

Center Utrecht, The Netherlands

${ }_{4}^{4}$ iMinds-Vision Lab, University of Antwerp, Belgium

Received 19 June 2017

accepted 10 October 2017

investigating the AF use a measure of white matter microstructural organization called Fractional Anisotropy (FA), which is limited by its inconsideration of voxels which include crossing fibres [10]. This study will employ a novel measure called hindrance modulated orientational anisotropy (HMOA) to study the $A F$, which addresses this limitation.

Reduced FA has been found bilaterally in the arcuate fasciculus in schizophrenia compared to healthy controls $[11,12]$, although more consistently in the left tract exclusively, and has been associated with auditory verbal hallucinations (AVHs) [13]. The cortical terminations of the arcuate fasciculus includes the inferior frontal gyrus, consisting of pars triangularis, pars orbitalis and pars opercularis; the inferior parietal cortex consisting of angular and supramarginal gyri; and the superior, middle and inferior temporal gyri [14]. Greater cortical thickness in frontal, temporal and 
parietal language regions have been linked to better verbal abilities in healthy adults [15, 16]. While reduced thickness and functional activation in these areas have been found in schizophrenia [17, 18, 19], evidence of cortical abnormalities has not been attributed directly to verbal deficits in psychosis. However, verbal performance has been associated with regions in the right hemisphere such as the inferior frontal gyrus and superior temporal gyrus in schizophrenia [17]. Therefore, this study aims to investigate if structural abnormalities are present in the AF and its associated cortical regions in individuals with psychosis and if these abnormalities are related to deficits of verbal cognitive performance in this group.

A secondary goal of the study is to examine asymmetry patterns of the AF network. The left hemisphere shows a prominence for language function $[17,20]$. The long segment of the $A F$ demonstrates a leftward asymmetry in healthy individuals [21, 22] and plays a dominant functional role in language [23]. The anterior AF tract has been found to predominantly present a rightward symmetry and a bilateral distribution is evident in the posterior segment [21]. Abnormalities in the asymmetry of the AF are suggested to occur in schizophrenia which may contribute to positive symptoms of the disorder [24] although findings are heterogeneous with some studies finding no abnormal laterality patterns [25]. Cortical asymmetries in the human brain are more subtle [26]. The most consistently reported left lateralized structure, the planum temporale (PT), [27, 28], is implicated in language function and synonymous with a portion of Wernicke's region. Despite heterogeneous findings due to methodological inconsistencies, predominantly the PT appears to have reduced leftward asymmetry in schizophrenia $[29,30]$ and has been associated with clinical symptoms [31]. Reduced left laterality has also been reported in the volume of the STG in schizophrenia and linked with cognitive deficits [19]. Therefore we aim to examine if reductions in the normal left asymmetry of the $A F$, and the language related cortical structures to which this tract projects to, contribute to verbal learning and verbal fluency deficits in psychosis. The majority of imaging studies investigating brain laterality employ measures of cortical surface area and cortical volume [29]. As cortical volume (CV) alone is not an optimal measure of asymmetry due it being a product of two independent measures, cortical thickness (CT) and surface area (CSA) [32], this study will combine these three indices of cortical measurement in its analysis (CT, CSA, CV) and to our knowledge is the first study to do so in examining laterality patterns in schizophrenia.

Finally, FA of the AF has been positively associated with cortical thickness in regions such as superior and middle temporal gyrus, supramarginal gyrus and inferior frontal gyrus in healthy controls, more pronounced in the left hemisphere [33].Therefore, we aim to extend this investigation in controls using $\mathrm{HMOA}$, and also investigate the relationship in individuals with psychosis.

In summary, using structural and diffusion MRI, the current study aims to investigate microstructural organization of three segments of the arcuate fasciculus and the cortical thickness, volume and surface area of seven of its cortical terminations, to determine whether abnormalities in the structures or in the laterality patterns of these brain regions contribute to impairments in verbal learning and verbal fluency in psychosis. As many brain abnormalities in schizophrenia are small and subtle in nature [34], segregated points along each AF segment will be additionally examined in relation to verbal cognition. Specifically, we hypothesise that 1) individuals with psychosis will have reduced HMOA/FA in the $\mathrm{AF}$ and reduced cortical thickness in left frontal, temporal and inferior parietal lobes compared to controls; 2) in healthy controls, verbal learning/fluency will be positively associated with microstructural organisation of the AF and its associated cortical regions, specifically in the left hemisphere, in contrast it is hypothesised that in the psychosis group verbal scores will be positively associated with right hemisphere regions of the AF network such as the inferior frontal and superior temporal gyri, with reference to previous literature [17]; 3) individuals with psychosis will have a reduction of the normal left laterality in the AF network evident in controls, particularly in the superior temporal gyrus; we also hypothesise that reduced left laterality in regions such as the STG will be significantly associated with verbal scores in the psychosis group; and finally, 4) in controls there will be a positive association between HMOA/FA of the $\mathrm{AF}$ and thickness of superior and middle temporal, supramarginal and inferior frontal gyri in the left hemisphere [33]. Exploratory analysis investigating the relationship between measures of the AF and cortical thickness for the psychosis group will be conducted. Uncovering neuroanatomical determinants of these deficits of verbal abilities in psychosis could further our understanding of cognitive impairments of the disorder and assist in targeted therapeutic strategies.

\section{Methods and materials Participants}

Twenty-six individuals with recent onset of psychotic illness and 27 healthy controls $(\mathrm{HC})$, participated in the study (Table 1). Participants underwent cognitive testing and MR scanning at first-presentation of illness and four years later (follow-up), the latter time-point, which
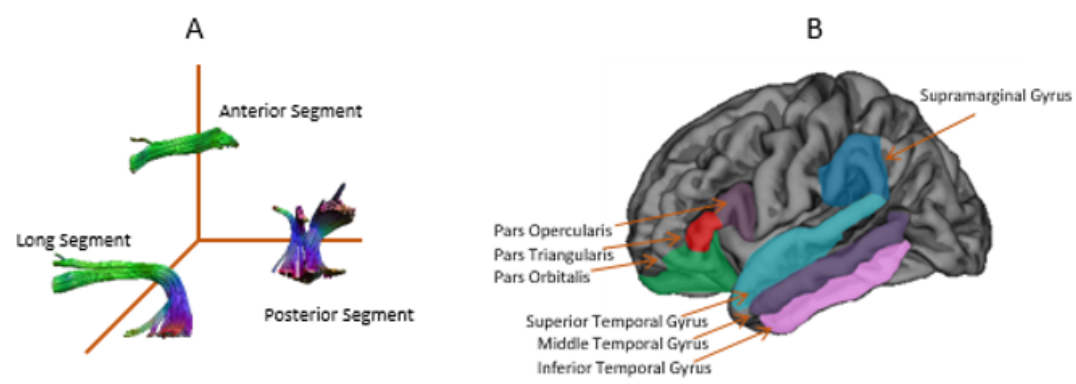

Figure 1: $(A)$ The three segments of the $A F$ and $(B)$ seven cortical regions where the arcuate fasciculus is known to project to 
included a diffusion tensor imaging acquisition, was used in the current analysis. All subjects were aged between 19 and 59 years. The recruitment and clinical assessment of these individuals are described previously [35, 36]. Exclusion criteria for all participants included neurological disorders (including epilepsy), comorbid substance or alcohol abuse in the last year, a history of head injury resulting in loss of consciousness for over $5 \mathrm{~min}$, oral steroid use in the previous 3 months and general contraindications to MRI. Healthy controls were also excluded if they had a personal or family history of psychotic or affective disorder. The study was approved by the research Ethics Committees of the National University of Ireland Galway and Galway University Hospitals. Written informed consent was obtained from all participants.

\section{Cognitive Measures}

The MATRICS Consensus Cognitive Battery (MCCB) was administered to individuals with psychosis and to a healthy control group. The MCCB assesses 7 cognitive domains known to be impaired in schizophrenia [37]. Two cognitive tests from the battery, verbal learning (Hopkins Verbal Learning Test -Revised) and verbal fluency (Category fluency: Animal fluency) were used in the current analyses (see [3] for description of cognitive tests).

\section{MRI Acquisition}

All subjects underwent structural MR and diffusion imaging at University Hospital Galway (UHG) in a 1.5 Tesla Siemens Magnetom Symphony scanner (Erlangen, Germany) equipped with a 4-channel
Table 1. Demographic characteristics and clinical features of the study sample

\begin{tabular}{|c|c|c|c|}
\hline & $\begin{array}{l}\text { Individuals } \\
\text { with psychosis }\end{array}$ & Healthy controls & Statistics $\left(t / x^{2}, p\right)$ \\
\hline $\mathrm{N}$ & 26 & 27 & \\
\hline Age (Mean yrs \pm SD) & $32 \pm 9$ & $38 \pm 9$ & $2.43,0.02^{*}$ \\
\hline Gender $N(m, f)(\%$ fem) & $16,10(31 \%)$ & $15,12(20 \%)$ & $0.20,0.70$ \\
\hline Education (Mean yrs $\pm S D$ ) & $16.1 \pm 2.8$ & $17.3 \pm 3.4$ & $1.43,0.16$ \\
\hline Handedness (left/right) & $4 / 22$ & $2 / 25$ & $8.4,0.36$ \\
\hline \multicolumn{4}{|l|}{ Diagnosis (N) } \\
\hline Schizophrenia & 7 & & \\
\hline Schizoaffective & 2 & & \\
\hline Psychosis NOS & 4 & & \\
\hline Mania & 10 & & \\
\hline Psychotic depression & 3 & & \\
\hline \multicolumn{4}{|l|}{ Medication (N) } \\
\hline Antipsychotics & 13 & & \\
\hline Mood stabilizers & 2 & & \\
\hline Antidepressants & 1 & & \\
\hline No medication & 7 & & \\
\hline Missing information & 2 & & \\
\hline CPZ equivalents @ FU & $202 \pm 281$ & & \\
\hline CPZ equivalents total & $245421 \pm 260750$ & & \\
\hline \multicolumn{4}{|l|}{ Symptoms: PANSS } \\
\hline Total score & $43.73 \pm 14.4$ & & \\
\hline Positive score & $10 \pm 3.65$ & & \\
\hline Negative score & $11.2 \pm 6.4$ & & \\
\hline General score & $22.6 \pm 6.3$ & & \\
\hline
\end{tabular}

Legend: $\mathrm{SD}=$ standard deviation, $\mathrm{T}=$ antipsychotic medication was converted to chlorpromazine equivalents Lehman et al. 1998; Taylor et al. 2007; Woods 2003); ${ }^{*}=$ significant difference between groups in age, $\mathrm{N}=$ number, PANSS = positive and negative symptom scale (0-6 per item scale version), FU = follow-up head coil. A volumetric T1-weighted magnetization-prepared rapid acquisition of gradient echo (MPRAGE) sequence was acquired with the imaging parameters: Repetition time (TR): $1140 \mathrm{~ms}$, echo time (TE): $4.38 \mathrm{~ms}$, inversion Time (TI): $600 \mathrm{~ms}$, flip angle 15; matrix size $256 \times 256$; an in-plane pixel size of $0.9 \mathrm{mmx} 0.9 \mathrm{~mm}$ and slice thickness of $0.9 \mathrm{~mm}$. Whole brain high angular resolution data was obtained in the axial orientation along 64 independent diffusion gradient directions, b-value $=1300 \mathrm{~s} / \mathrm{mm}^{2}$, with 7 reference non-diffusion-weighted images (b-value $\left.=0 \mathrm{~s} / \mathrm{mm}^{2}\right)$. Image parameters were: echo time $(T E)=95 \mathrm{~ms}$, repetition time (TR) $=8100 \mathrm{~ms}$, flip angle $=15^{\circ}$, voxel size $=2.5$ $\mathrm{mm}^{3}$, matrix size $=96 \times 96$, slice thickness $=$ $2.5 \mathrm{~mm}$, in-plane resolution $=2.5 \mathrm{~mm}^{2}$.

\section{Image processing and Quality \\ Assessment - Diffusion Data}

Explore DTI (version 4.8.4) was used to correct for motion and eddy current induced geometric distortions including rotation of the b-matrix to preserve the orientation information [38, 39]. Data quality was further assessed by visual inspection [40]. Whole brain tractography was carried out in ExploreDTI using the constrained spherical deconvolution (CSD) tracking algorithm [41] with recursive calibration of the response function [42]. The three segments of the $A F$, long, anterior and posterior, were isolated based on a strict anatomical protocol [21] by manually placing AND and NOT gates on directionally encoded colour anisotropy (DEC-FA) maps (See Supplementary material 1 for protocol description). Median HMOA and median $\mathrm{FA}$ of the bilateral three AF segments were extracted. Tract volume was covaried for when analyzing the FA of the segments. Additional tract resampling of the three segments was performed at a number of points over the length of each tract (mean length of tract divided by voxel size). The long tract was subdivided into 32 sections, the anterior tract into 23 sections and the posterior tract into 14 sections. Median HMOA and median FA were extracted at each section (see supplementary material 4) . 
Image Processing and Quality Assessment- Structural Data Intensity inhomogeneities in the T1-weighted images were corrected using nonparametric, non-uniform intensity normalization (N3) [43]. Image processing was carried out using FreeSurfer, stable version 5.1 (https://surfer. nmr.mgh.harvard.edu). Detailed descriptions of this method have already been reported $[35,44,45]$, but are briefly summarized in Supplementary material 2.

\section{Statistics}

All statistics were conducted using IBM SPSS [46]. ANCOVAs were used to identify any group differences in the three tracts of the arcuate fasciculus (median HMOA of the segment and at each point along each segment) and in the cortical thickness (CT), surface area (CSA) and volume (CV) of cortical regions bilaterally. Kruskal-Wallis tests were administered for any points along each $\mathrm{AF}$ segment that were not normally distributed. Partial correlation analyses were implemented for the psychosis group and the healthy controls when investigating the relationship between the $\mathrm{AF}$ and cortical regions with verbal learning and verbal fluency, and additionally when examining the association between $\mathrm{AF}$ anisotropy and cortical regions.

For each AF segment and cortical region (CT, CSA, CV) a lateralization index (LI) was calculated according to the formula (e.g. for HMOA: left HMOA-right HMOA/left HMOA-right HMOA). A positive value indicated a leftward asymmetry and a negative value indicated a rightward asymmetry. The degree of lateralization was determined using a one-sample t-test. ANCOVA models were implemented when investigating group differences in LI. For non-normally distributed LIs, non-parametric Mann-Whitney tests were conducted.

To correct for multiple comparisons, false discovery rate (FDR) correction was applied for all analyses. Left-handed individuals were removed ( $n=6$; Table 1$)$, with age and gender being covaried for in all analyses. Shapiro-Wilks tests were used to test for normal distribution of each variable investigated. The majority of variables were normally distributed with any non-normal distributions successfully transformed (See Supplementary material 3). As the most novel measure of white matter organization, $\mathrm{HMOA}$, is not a well-established measure of microstructural organisation in the literature, it is presented along a conventional index, FA, in this paper. FA of the arcuate fasciculus was investigated post-hoc and results are included in Supplementary Material 5.

\section{Results}

\section{Group differences in verbal learning and verbal fluency}

The individuals with psychosis had significantly reduced verbal learning (VL) and verbal fluency (VF) scores compared to healthy controls (Figure 2A)

\section{Group Differences in the Arcuate Fasciculus and Relationship to Verbal} Cognition

HMOA: There were no significant differences between the individuals with psychosis and controls in the median HMOA of the long, anterior or posterior segments $(F=0.01$ $3.59, \mathrm{p}=0.10-0.91)$, nor at any individual points A
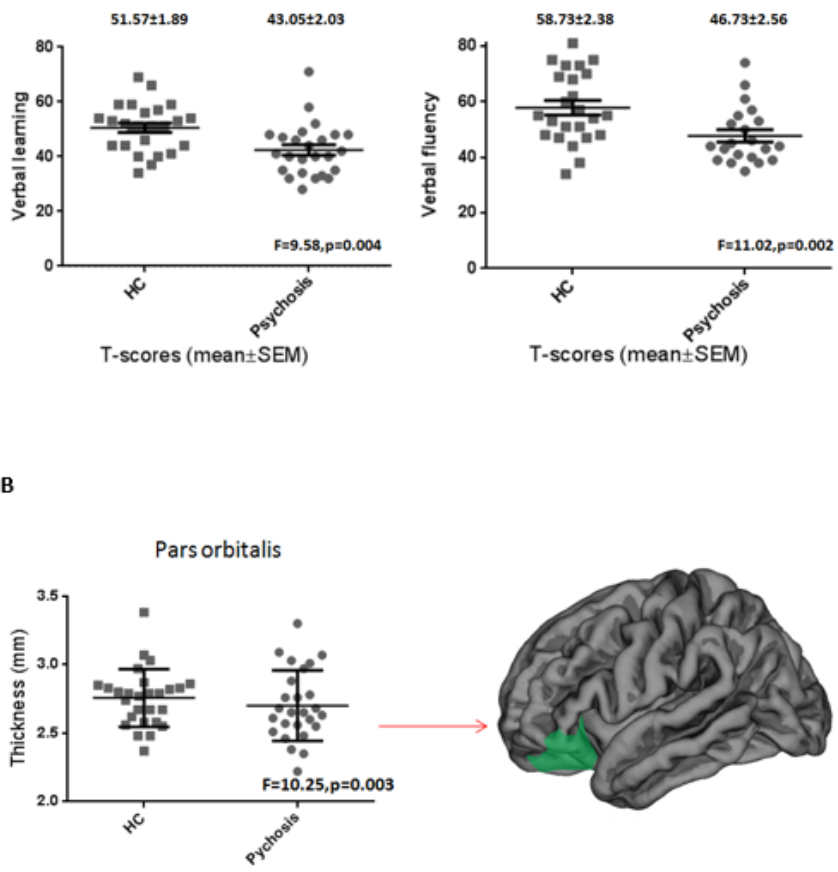

Figure 2: (A) Individuals with psychosis performed significantly poorer on verbal learning and verbal fluency compared to healthy controls $(\mathrm{HC})$; $(\mathrm{B})$ Individuals with psychosis had lower cortical thickness compared to healthy controls $(\mathrm{HC})$ in the right hemisphere pars orbitalis which is part of the inferior frontal gyrus 
in the pars orbitalis (mean $\pm \mathrm{SE}: 2.63 \pm 0.04$ ) compared to controls (mean $\pm \mathrm{SE}: 2.83 \pm 0.04$ ), $(F=10.25, p=0.003)$ (Figure 2B). There were no further significant group differences, surviving multiple comparisons correction, in the thickness, surface area or volume of any cortical regions $(\mathrm{F}=0.03-6.68, \mathrm{p}=0.013-0.87)$.

Lower surface area $(r=-0.61, p=0.003)$ and volume $(r=-0.60, p=0.003)$ of the right superior temporal gyrus (STG) was significantly associated with greater verbal fluency performance in the control group (Figure $3 A, B)$. These associations were in the opposite direction to what was stated in hypothesis 2 , present in the right hemisphere only and not the left as hypothesised. In the psychosis group, greater volume in the right pars orbitalis was significantly associated with greater verbal fluency $(r=0.62, p=0.004) \quad$ (Figure $3 C)$, while greater volume in the right pars opercularis was significantly related to better verbal learning scores ( $r=0.64, p=0.003$ ) (Figure 3D), in support of hypothesis 2. No other significant correlations were found between the thickness, surface area and volume of any other cortical regions and verbal learning ( $r=-0.23-0.36, p=0.02-0.98)$ or verbal fluency $(r=-0.46-0.22, p=0.02-0.99)$.
Patterns of Lateralization of the Arcuate Fasciculus and Relationship to Verbal Cognition

HMOA: In the healthy controls, the lateralization index (LI-HMOA) of the long and posterior segment displayed significant leftward asymmetry patterns, while the anterior segment was significantly rightward in its symmetry (Figure 4). In the individuals with psychosis, a leftward asymmetry was present in the long segment, with no significant asymmetry patterns evident in the anterior or posterior segments (Figure 4). Individuals with psychosis and healthy controls did not significantly differ in the laterality patterns of the three $\mathrm{AF}$ segments $(F=0.42-3.37, p=0.08-0.52)$. When investigating the relationship with cognition, there were no significant associations between the asymmetry of the three AF segments and verbal learning or verbal fluency (Figure 4).

FA: When investigating laterality with measures of FA (LI-FA), the magnitude and direction of results were similar to $\mathrm{HMOA}$, with the exception of no significant asymmetry reported in the posterior segment of the healthy controls. Similar to HMOA, no group differences in laterality were reported and laterality did not significantly relate to cognition (Supplementary 5C).

\section{Patterns of Lateralization of the} Associated Cortices of the Arcuate Fasciculus and Relationship to Verbal

\section{Cognition}

Investigating laterality patterns of cortical thickness revealed a significant leftward asymmetry in the inferior temporal gyrus of the healthy controls. Individuals with psychosis demonstrated no significant asymmetry patterns in the thickness of any cortical regions (Supplementary 6). Investigating cortical surface area (CSA) revealed a significant leftward asymmetry in the pars opercularis, STG and ITG, and a significant rightward asymmetry in the pars triangularis, pars orbitalis and MTG. Individuals with psychosis demonstrated similar directions of cortical symmetry to controls with the exception of no significant STG asymmetry. CV laterality revealed identical symmetry patterns to CSA in controls and similar symmetry patterns to CSA in individuals with psychosis excepting differences in the STG and ITG (Supplementary 6).

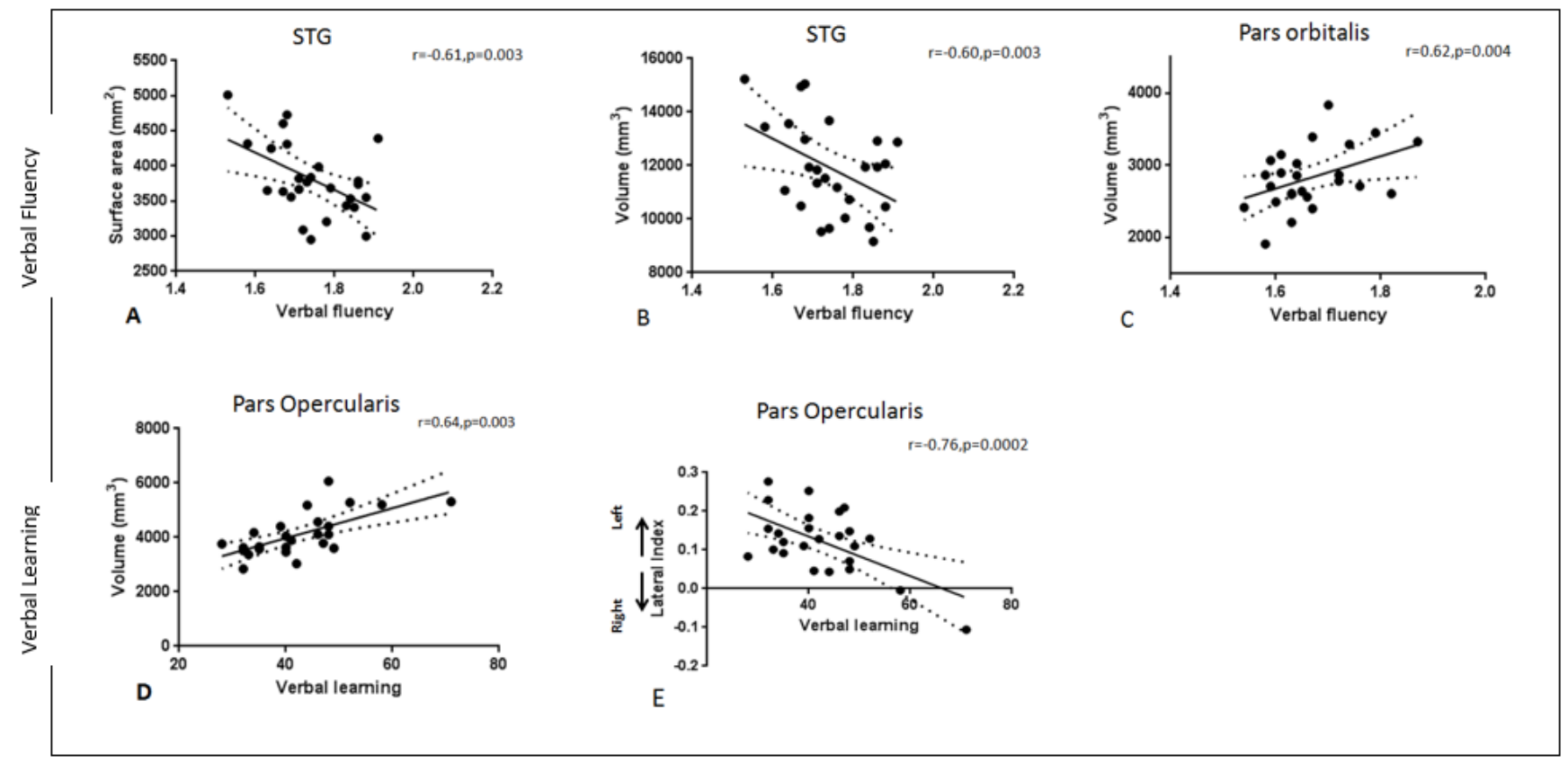

Figure 3: Reduced surface area (A) and volume (B) in the right STG was correlated with better performance on a verbal fluency task (log transformed) in healthy controls. For the individuals with psychosis, better verbal fluency performance was related to greater volume in the right pars orbitalis (C) while greater volume in the right pars opercularis (D) and reduced left lateralization (volume) of the pars opercularis (E) was associated with better verbal learning performance 
Not in support of hypothesis 3, individuals with psychosis and controls did not significantly differ in the LI of any cortical regions (thickness, surface area or volume) (Supplementary 7). When investigating the relationship with cognition, we found a relationship between reduced left lateralization of the volume of the pars opercularis and better verbal learning $(r=-0.76, p=0.0002)$ (Figure 3E) in the psychosis group, although no association was found in the STG as hypothesised. There were no other significant relationships between laterality and cognition in both groups (VL: $r=-0.59$ $0.41, p=0.008-0.89 ; \mathrm{VF}: \mathrm{r}=-0.44-0.47, \mathrm{p}=0.04-96$ ).

\section{Relationship between the Arcuate Fasciculus and Its Associated \\ Cortices}

HMOA: There were no significant relationships between any of the three AF segments and cortical regions (thickness, surface area or volume) in either group ( $r=-0.29$ $0.50, p=0.03-0.98$ ).

FA: When investigating measures of $F A$, significant associations were identified in the control group between the left anterior segment and the left pars orbitalis thickness $(r=-0.60, p=0.003)$ and the left supramarginal gyrus thickness $(r=-0.59, p=0.004)$ in support of hypothesis 4 . No other significant associations were found in either group which survived multiple comparisons correction ( $r=-0.43$ $0.59, p=0.008-0.98$ ).

\section{Discussion}

The current study, which included a comprehensive investigation of the arcuate fasciculus (AF) language network in relation to verbal cognitive deficits in psychosis, identifies findings predominantly in the neuroanatomy of the right hemisphere of this network, which may contribute to cognitive impairments. Microstructural organisation of the right long segment of the $A F$, as indexed by $F A$, is related to verbal fluency performance in individuals with psychosis. This relationship was not evident in healthy controls and may be indicative of a pathological role of this tract in verbal fluency performance. Previously, the right AF has been identified as a potential trait marker for schizophrenia and has shown trends of a relationship with positive symptoms [47]. The magnitude and direction of results for HMOA of the right long $A F$ was consistent with $F A$, though not statistically significant. In relation to cortical regions, the psychosis group revealed a positive association between cortical volume in the right pars opercularis and pars orbitalis and performance in VL and VF respectively. Additionally, reduced left asymmetry or increased right asymmetry in the volume of the pars opercularis is associated with better VL in psychosis. Combined, our findings may implicate an abnormal involvement of right inferior frontal gyri cortical regions in verbal tasks in psychosis, which the right long $\mathrm{AF}$ tract projects to, as in healthy individuals this region plays a limited role in language lacking any phonological or semantic representation, with the right pars opercularis specifically activated by tonal and pseudowords [48]. Additionally, we report reduced thickness in the right pars orbitalis in the psychosis group, which has previously been reported in early stages of the illness [14].

In healthy controls, a reduction in the right hemisphere superior temporal gyrus (STG) volume and surface area is associated with greater verbal fluency performance, not present in the psychosis group. PET and FMRI studies show that verbal fluency tasks activate frontal regions coupled with a deactivation in bilateral STG $[49,50]$ with a failure of STG deactivation suggested to occur in schizophrenia $[50,51]$. The absence of reduced STG volume involvement in verbal fluency performance in the psychosis group may indicate pathology in this cortical region contributing to impairments on this task. However, it is uncertain precisely how functional activation of the STG relates to volume of the cortex. An alternative interpretation may be that normal synaptic pruning in the STG which results in greater vocabulary specialization [52] may correspondingly not occur in individuals with psychosis. One previous study reported increased performance in verbal fluency to

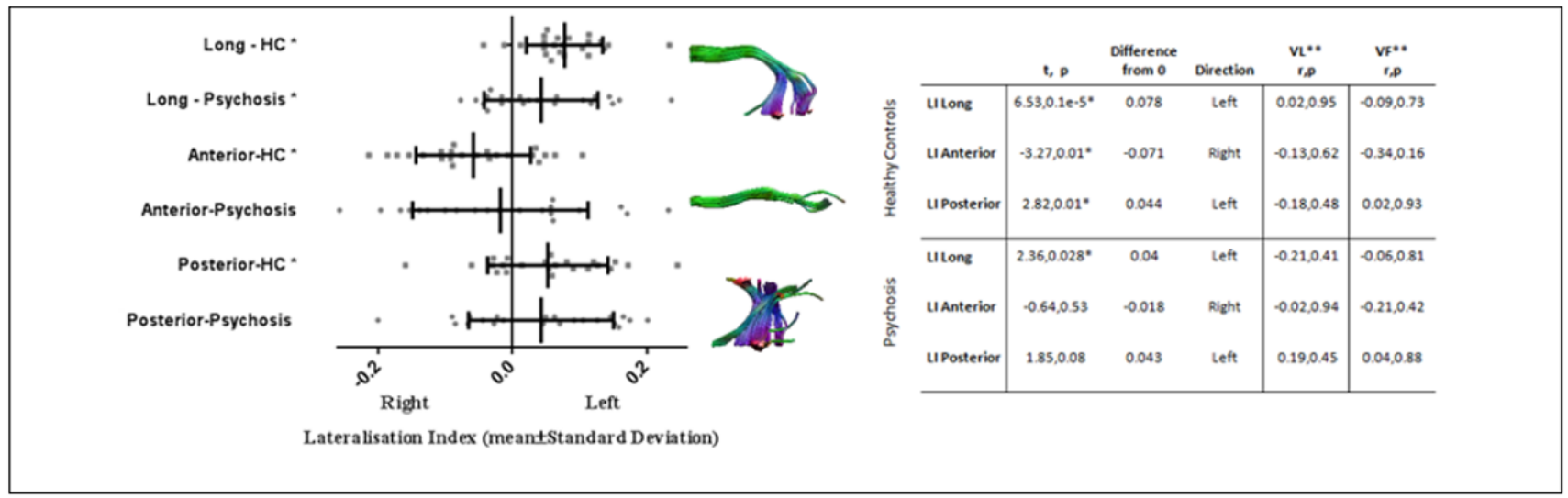

Figure 4: Laterality patterns of the long, anterior and posterior segments of the arcuate fasciculus in healthy controls (HC) and individuals with psychosis. ${ }^{*}=$ significant laterality patterns in the arcuate fasciculus; $\mathrm{HC}=$ healthy control; $\mathrm{LI}=$ lateralization index; ${ }^{* *}=$ relationship of $\mathrm{LI}$ to verbal learning and verbal fluency, covarying for age and gender. 
be associated with decreased thickness in bilateral STG, among other language regions [53].

Distinct patterns of laterality in the three segments of the AF are evident. In healthy controls, we find a left asymmetry in the long and posterior segment, whereas the anterior segment was rightward lateralized, consistent with previous research excepting the posterior segment which was previously reported to be bilateral [21]. A leftward asymmetry was found in the long segment in the psychosis group. Analyses of cortical surface area and cortical volume reveals similar patterns of asymmetry in multiple regions consistent with previous studies [32]. Analysis of cortical thickness reveals a significant leftward pattern in the inferior temporal gyrus in controls only. The current study does not report any abnormal laterality patterns in the psychosis group in the arcuate fasciculus or associated cortical regions. Previous studies indicate slight attenuation in the normal lateralization pattern in schizophrenia [54], however studies regarding the arcuate fasciculus and cortical asymmetry are heterogeneous, with some studies showing no differences between patients and controls $[25,55,56$, 57]. While the planum temporale (PT) is the most consistently reported region exhibiting abnormal asymmetry in schizophrenia, this study does not investigate the PT explicitly but rather the entire STG region which may have obscured any true abnormal asymmetry in the PT.

Contrasting to other studies where abnormal increases or decreases in FA of the $A F$ have been found in schizophrenia $[58,11]$, this study fails to identify any abnormalities in $\mathrm{HMOA}$ or $\mathrm{FA}$ of the $\mathrm{AF}$ in individuals with psychosis relative to controls. Variability of findings may be due to differences in the anatomical definitions of the AF employed in studies and the fact that the body of the three AF segments are examined only in the current study, excluding tract endings to reduce variance. The current study does not report any relationship between HMOA of the three AF segments and its associated cortical regions in contrast to other studies using FA [33]. However, post-hoc analysis of FA measures uncovers a relationship between the left $A F$ and left frontal and parietal cortical regions in the control group.

The importance of investigating language related brain regions and verbal deficits in schizophrenia is highlighted by the relationship between verbal cognition and auditory verbal hallucinations (AVHs) [59] which may share similar neural substrates, however this was not replicated in the current paper (Supplementary 8). While in general there is more support for a relationship between cognition and negative symptoms compared to positive symptoms, deficits in verbal cognitive tasks and abnormalities in the brain networks involved may be potential markers of development of auditory verbal hallucinations (AVH) [5].

Our findings mainly suggest a possible dysfunctional role of the right hemisphere, as significant findings were present in the right long $A F$ segment and right frontal and temporal cortices, areas to which the long segment projects to. AVHs have been associated with activation of the right inferior frontal area [60] and to impaired connectivity in fronto-temporal language regions [61] therefore our findings could support the involvement of fronto-temporal cortical abnormalities in language function and potentially psychotic symptoms in psychosis. Post-hoc analysis revealed that our finding of an association between asymmetry of the pars opercularis and verbal learning in the psychosis group was driven by those experiencing AVHs (Supplementary 8). Sample size and consequently statistical power was low, therefore it is recommended that future studies explicitly investigate the role of abnormal cortical asymmetry in auditory verbal hallucinations.

Strengths of the current study include a comprehensive exploration of the AF using a novel measure of anisotropy (HMOA) and a detailed examination of the language cortical regions using three indices of grey matter architecture, in relation to verbal deficits in psychosis. Limitations of the study include the limited sample size and clinical heterogeneity of the sample and the use of directionally encoded colour anisotropy (DEC-FA) maps when isolating the arcuate fasciculus. As the response function of the HMOA, which represents the diffusion signal profile of white matter fibre orientation, is individually optimised, it is unclear what effect nonnormalisation of the response function has on findings in the current study and future investigation into this is warranted. Also, the correlational nature of the analysis renders it possible only to infer associations between the language network studied and verbal cognitive measures.

\section{Conclusion}

In summary, our findings suggest a possible dysfunctional role of the right hemispheric arcuate fasciculus network, including the right long arcuate fasciculus white matter tract and right inferior frontal and superior temporal cortices, in verbal learning and verbal fluency impairments in psychosis. Abnormal laterality in the pars opercularis may also contribute to verbal learning deficits in psychosis, thereby further implicating an atypical role of the right inferior frontal gyrus in verbal cognitive tasks in psychotic illness.

\section{Acknowledgements}

We would like to thank the participants for their time and effort. This work was supported by the Health Research Board (HRA_POR/2011/100) and the Irish Research Council (GOIPG/2013/672) (IRCHSS) PhD Scholarship (JK). BJ is a postdoctoral fellow of the Research Foundation - Flanders (FWOVlaanderen). An abstract using this data was previously published in 2016 in European Neuropsychopharmacology, 26:76-77.

\section{Financial Disclosures}

The authors declare no financial interests or potential conflicts of interest 
[1] Aas M., Dazzan P., Mondelli V., Melle I., Murray R.M., Pariante C.M., A systematic review of cognitive function in first-episode psychosis, including a discussion on childhood trauma, stress, and inflammation, Front. Psychiatry., 2014, 4, 1-13.

[2] Bozikas V.P., Andreou C., Longitudinal studies of cognition in first episode psychosis: a systematic review of the literature, Aust. N. Z. J. Psychiatry., 2011, 45, 93-108.

[3] Kenney J., Anderson-Schmidt H., Scanlon C., Arndt S., Scherz E., Mclnerney $S_{\text {., }}$ et al., Cognitive course in first-episode psychosis and clinical correlates: A 4 year longitudinal study using the MATRICS Consensus Cognitive Battery, Schizophr. Res., 2015, 169, 101-108.

[4] Lin A., Wood S.J., Nelson B., Brewer W.J., Spiliotacopoulos D., Bruxner A., et al., Neurocognitive predictors of functional outcome two to 13 years after identification as ultra-high risk for psychosis, Schizophr. Res., 2011, 132, 1-7.

[5] Lencz T., Smith C.W., McLaughlin D., Auther A., Nakayama E., Hovey L., Cornblatt B.A., Generalized and specific neurocognitive deficits in prodromal schizophrenia, Biol. Psychiatry., 2006, 59, 863-871.

[6] Klosterkotter J., Hellmich M., Steinmeyer E.M., Schultze-Lutter F., Diagnosing Schizophrenia in the Initial Prodromal Phase, Arch. Gen. Psychiatry., 2016, 58, 158-164.

[7] De Weijer A.D., Neggers S.F.W., Diederen K.M.S., Mandl R.C.W., Kahn R.S., Hulshoff Pol H.E., Sommer I.E., Aberrations in the arcuate fasciculus are associated with auditory verbal hallucinations in psychotic and in non-psychotic individuals, Hum. Brain Mapp., 2013, 34, 626- 634 .

[8] Wernicke C., Der aphasische symptomen complex: eine psychologische studie auf anatomischer basis, In: Eggert GH (Ed.), Wernicke's Works on Aphasia: A Sourcebook and Review: Mouton, 1874/1977, 91-145.

[9] Catani M., Jones D.K., Ffytche D.H., Perisylvian language networks of the human brain, Ann. Neurol., 2005, 57, 8-16.

[10] Dell'Acqua F., Simmons A., Williams S.C.R., Catani M., Can spherical deconvolution provide more information than fibre orientations? Hindrance modulated orientational anisotropy, a true-tract specific index to characterize white matter diffusion, Hum. Brain Mapp., 2013, 34, 2464-83.

[11] Shergill S.S., Kanaan R.A., Chitnis X.A., O'Daly O., Jones D.K., Frangou S., et al., A Diffusion Tensor Imaging Study of Fasciculi in Schizophrenia, Am. J. Psychiatry., 2007, 164, 467-473

[12] Catani M., Craig M.C., Forkel S.J., Kanaan R., Picchioni M., Toulopoulou T., et al. Altered integrity of perisylvian language pathways in schizophrenia: relationship to auditory hallucinations, Biol. Psychiatry., 2011, 70, 1143-1150.

[13] De Weijer A.D., Mandl R.C.W., Diederen K.M.J., Neggers S.F.W., Kahn R.S., Hulshoff Pol H.E., et al., Microstructural alterations of the arcuate fasciculus in schizophrenia patients with frequent auditory verbal hallucinations, Schizophr. Res., 2011, 130, 68-77.

[14] Francis A.N., Seidman L.J., Jabbar G.A., Mesholam-Gately R., Thermenos H.W., Juelich R., et al., Alterations in brain structures underlying language function in young adults at high familial risk for schizophrenia, Schizophr. Res., 2012, 141, 65-71.

[15] Hartberg C.B., Lawyer G., Nyman H., Jönsson E.G., Haukvik U.K., Saetre P., et al., Investigating relationships between cortical thickness and cognitive performance in patients with schizophrenia and healthy adults, Psychiatry Res., 2010, 182, 123-133.

[16] Antonova E., Kumari V., Morris R., Halari R., Anilkumar A., Mehrotra R., et al., The relationship of structural alterations to cognitive deficits in schizophrenia: a voxel-based morphometry study, Biol. Psychiatry., 2005, 58, 457-467.

[17] Ehrlich S., Brauns S., Yendiki A., Ho B.C., Calhoun V., Schulz S.C., et al., Associations of cortical thickness and cognition in patients with schizophrenia and healthy controls, Schizophr. Bull., 2012, 38, 1050-1062.

[18] Jeong B., Wible C.G., Hashimoto R., Kubicki M., Functional and anatomical connectivity abnormalities in left inferior frontal gyrus in schizophrenia, Hum. Brain Mapp., 2009, 30, 4138- 4151.

[19] Vita A., Dieci M., Giobbio G.M., Caputo A., Comazzi M., Garbarini $M$., et al., Language and thought disorder in schizophrenia : brain morphological correlates, Schizophr. Res., 1995, 15, 243-251.

[20] Bethmann A., Tempelmann C., De Bleser R., Scheich H., Brechmann A., Determining language laterality by $\mathrm{fMRI}$ and dichotic listening, Brain Res., 2007, 1133, 145-157.

[21] Catani M., Allin M.P.G., Husain M., Pugliese L., Mesulam M.M., Murray R.M., et al., Symmetries in human brain language pathways correlate with verbal recall, Proc. Natl. Acad. Sci. U.S.A., 2007, 104, 1716317168.

[22] Vernooij M.W., Smits M., Wielopolski P.A., Houston G.C., Krestin G.P., Van der Lugt A., Fibre density asymmetry of the arcuate fasciculus in relation to functional hemispheric language lateralization in both right- and left-handed healthy subjects: a combined fMRI and DTI study, Neurolmage., 2007, 35, 1064-1076.

[23] Vassal F., Schneider F., Boutet C., Jean B., Sontheimer A., Lemaire J.J., Combined DTI Tractography and Functional MRI Study of the Language Connectome in Healthy Volunteers: Extensive Mapping of White Matter Fascicles and Cortical Activations, PloS One., 2016, 11, 1-19.

[24] Abdul-Rahman MF., Qiu A., Woon P.S., Kuswanto C., Collinson S.L., Sim K., Arcuate fasciculus abnormalities and their relationship with psychotic symptoms in schizophrenia, PloS One., 2012, 7, 1-9.

[25] Miyata J., Sasamoto A., Koelkebeck K., Hirao K., Ueda K., Kawada R., et al., Abnormal asymmetry of white matter integrity in schizophrenia revealed by voxelwise diffusion tensor imaging, Hum. Brain Mapp., 2012, 33, 1741-1749.

[26] Crow T.J., Cerebral asymmetry and the lateralization of language: core deficits in schizophrenia as pointers to the gene, Curr. Opin. Psychiatry., 2004, 17, 97-106.

[27] Shapleske J., Rossell S.L., Simmons A., David A.S., Woodruff P.W.R., Are Auditory Hallucinations the Consequence of Abnormal Cerebral Lateralization? A Morphometric MRI Study of the Sylvian Fissure and Planum Temporale, Biol. Psychiatry., 2001, 3223, 685-693. 
[28] Harasty J., Seldon H.L., Chan P., Halliday G., Harding A., The left human speech-processing cortex is thinner but longer than the right, Laterality., 2003, 8, 247-260.

[29] Shapleske J., Rossell S.L., Woodruff P.W.R., David A.S., The planum temporale: a systematic, quantitative review of its structural, functional and clinical significance, Brain Res. Rev., 1999, 29, 26-49.

[30] Hasan A., Kremer L., Gruber O., Schneider-Axmann T., Guse B., Reith W., et al., Planum temporale asymmetry to the right hemisphere in first-episode schizophrenia, Psychiatry Res., 2011, 193, 56-59.

[31] Oertel-Knöchel V., Linden D.E.J., Cerebral asymmetry in schizophrenia, Neuroscientist., 2011, 17, 456-467.

[32] Meyer M., Liem F., Hirsiger S., Jäncke L., Hänggi J., Cortical surface area and cortical thickness demonstrate differential structural asymmetry in auditory-related areas of the human cortex, Cereb. Cortex., 2014, 24, 2541-52.

[33] Phillips O.R., Clark K.A., Woods R.P., Subotnik K.L., Asarnow R.F., Nuechterlein K.H., et al., Topographical relationships between arcuate fasciculus connectivity and cortical thickness, Hum. Brain Mapp., 2011, 32, 1788-801.

[34] Shenton M.E., Dickey C.C., Frumin M., Mccarley R.W., A review of MRI findings in schizophrenia, Schizophr. Res., 2001, 49, 1-52.

[35] Scanlon C., Anderson-Schmidt H., Kilmartin L., Mclnerney S., Kenney J., McFarland J., et al., Cortical thinning and caudate abnormalities in first episode psychosis and their association with clinical outcome, Schizophr. Res., 2014, 159, 36-42.

[36] McFarland J., Cannon D.M., Schmidt H., Ahmed M., Hehir S., Emsell L., et al., Association of grey matter volume deviation with insight impairment in first-episode affective and non-affective psychosis, Eur. Arch. Psychiatry Clin. Neurosci., 2013, 263, 133-141.

[37] Kern R.S., Nuechterlein K.H., Green M.F., Baade L.E., Fenton W.S., Gold J.M., et al., The MATRICS Consensus Cognitive Battery, part 2: conorming and standardization, Am. J. Psychiatry., 2008, 165, 214-220.

[38] Leemans A., Jeurissen B., Sijbers J., Jones D.K., "ExploreDTI: A graphical toolbox for processing, analyzing, and visualizing diffusion MR data", Proceedings of the 17th Scientific Meeting, International Society for Magnetic Resonance in Medicine (Honolulu, USA) 2009a, 3537.

[39] Leemans A., Jones D.K., The $B$-matrix must be rotated when correcting for subject motion in DTI data, Magn. Reson. Med., 2009b, 61, 13361349.

[40] Tournier J.D., Mori S., Leemans A., Diffusion tensor imaging and beyond, Magn. Reson. Med., 2011, 65, 1532-1556.

[41] Jeurissen B., Leemans A., Jones D.K., Tournier J.D., Sijbers J., Probabilistic fibre tracking using the residual bootstrap with constrained spherical deconvolution, Hum. Brain Mapp., 2011, 32, 461-479.

[42] Tax C.M.W., Jeurissen B., Vos S.B., Viergever M.A., Leemans A., Recursive Calibration of the fibre response function for spherical deconvolution of diffusion MRI data, Neuroimage., 2013, 86, 6780.

[43] Sled J.G., Zijdenbos A.P., Evans A.C., A nonparametric method for automatic correction of intensity nonuniformity in MRI data, Trans. Med. Imaging., 1998, 17, 87-97.
[44] Dale A.M., Fischl B., Sereno M.l., Cortical surface-based analysis: I. Segmentation and surface reconstruction, Neuroimage., 1999, 9, 179-194.

[45] Fischl B., Sereno M.I., Dale A.M., Cortical surface-based analysis II: inflation, flattening, and a surface-based coordinate system, Neuroimage., 1999, 9, 195-207.

[46] Statistics for Windows, Version 21.0. Armonk, NY: IBM Corp.

[47] Wu C.H., Tzung-Jen H., Chen Y.J., Hsu Y.C., Lo Y.C., Liu C.M., Altered integrity of the right arcuate fasciculus as a trail marker of schizophrenia: A sibling study using tractography-based analysis of the whole brain, Hum. Brain Mapp., 2014, 36, 1065-1076.

[48] Vigneau M., Beaucousin V., Hervé P.Y., Jobard G., Petit L., Crivello F., et al., What is right-hemisphere contribution to phonological, lexicosemantic, and sentence processing? Insights from a meta-analysis, Neurolmage., 2011, 54, 577-593.

[49] Schlösser R., Hutchinson M., Jose S.V., Rusinek H., Saarimaki A., Stevenson J., et al., Functional magnetic resonance imaging of human brain activity in a verbal fluency task, J. Neurol. Neurosurg. Psychiatry., 1998, 64, 492-498.

[50] Frith C.D., Friston S., Herold D., Silbersweig P., Fletcher P., Cahill C., et al., Regional brain activity in chronic schizophrenic patients during the performance of a verbal fluency task, Br. J. Psychiatry., 1995, 167, 343-349.

[51] Fletcher P.C., Frith C.D., Grasby P.M., Friston K.J., Dolan R.J., Unit C., et al., Local and Distributed Effects of Apomorphine on Fronto-Temporal Function in Acute Unmedicated Schizophrenia, J. Neurosci., 1996, 16, 7055-7062.

[52] Sowell E.R., Thompson P.M., Leonard C.M., Welcome S.E., Kan E., Toga A.W., Longitudinal mapping of cortical thickness and brain growth in normal children, J. Neurosci., 2004, 24, 8223-8231.

[53] Porter J.N., Collins P.F., Muetzel R.L., Lim K.O., Luciana M., Associations between cortical thickness and verbal fluency in childhood, adolescence, and young adulthood, Neurolmage., 2011, 55, 1865-1877.

[54] Park H.J., Westin C.F., Kubicki M., Maier S.E., Niznikiewicz M., Baer A., et al., White matter hemisphere asymmetries in healthy subjects and in schizophrenia: a diffusion tensor MRI study, Neurolmage., 2004, 23, 213-223.

[55] Hamilton L.S., Narr K.L., Luders E., Szeszko P.R., Thompson P.M., Bilder R.M., et al., Asymmetries of cortical thickness: effects of handedness, sex, and schizophrenia, Neuroreport., 2007, 18, 1427-1431.

[56] Takao H., Abe O., Yamaue H., Aoki S., Kasai K., Ohtomo K., Cerebral asymmetry in patients with schizophrenia: A voxel-based morphometry (VBM) and diffusion tensor imaging (DTI) study, J. Magn. Reson. Imaging., 2010, 31, 221-226.

[57] Meisenzahl E.M., Frodl T., Müller D., Schmitt G., Gallinat J., Zetzsche T., et al., Superior temporal gyrus and P300 in schizophrenia: a combined ERP/structural magnetic resonance imaging investigation, J. Psychiatr. Res., 2004, 38, 153-162

[58] Rotarska-Jagiela A., Oertel-Knoechel V., DeMartino F., van de Ven V., Formisano E., Roebroeck A., et al., Anatomical brain connectivity and positive symptoms of schizophrenia: a diffusion tensor imaging study, Psychiatry Res., 2009, 174, 9-16. 
[59] Gisselgård J., Anda L.G., Brønnick K., Langeveld J., Ten Velden Hegelstad W., Joa I., et al., Verbal working memory deficits predict levels of auditory hallucination in first-episode psychosis, Schizophr. Res., 2014, 153, 38-41.

[60] Sommer I.E.C., Diederen K.M.J., Blom J.D., Willems A., Kushan L., Slotema K., et al., Auditory verbal hallucinations predominantly activate the right inferior frontal area, Brain., 2008, 131, 3169-3177. [61] Curcic-Blake B., Liemburg E., Vercammen A., Swart M., Knegtering

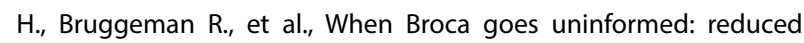
information flow to Broca's area in schizophrenia patients with auditory hallucinations, Schizophr. Bull., 2013, 39, 1087-1095. 